\title{
Effect of Exericse and Nutrition upon Lifestyle-Related Disease and Cognitive Function
}

\author{
Toshio MORITANI and Yasunori AKAMATSU \\ Graduate School of Human and Environmental Studies, Kyoto University, \\ Sakyo-ku, Kyoto 606-8501 Japan
}

\begin{abstract}
Summary Bray has proposed the "MONA LISA" hypothesis, an acronym for Most Obesities kNown Are Low In Sympathetic Activity indicating that obesity is associated with a relative or absolute reduction in the activity of the thermogenic component of the sympathetic nervous system. Our series of studies have suggested a potential reversibility in ANS activity regulating fat metabolism and appetite control by regular exercise training in middle aged individuals and obese children with depressed ANS activity. In other words habitual exercise plays a vital role in enhancing not only fat and glucose metabolism, but also ANS activities in the prevention of obesity and appetite control. There are growing expectations that too much sitting is a real and substantial risk to health. One of the intriguing findings from these accelerometer measurement studies is that breaks in sedentary time were shown to have beneficial associations with metabolic biomarkers, due possibly to challenging and enhancing autonomic nervous system that regulates body weight and appetite. Recent findings of brain-derived neurotrophic factors (BDNF) seem to have shed some light upon agerelated neurodegenerative diseases and appear to influence energy metabolism, appetite and aspects of neuro-cognitive function. These data strongly suggest that a lack of exercise as characterized by a sedentary lifestyle and an unhealthy diet may lead to accelerated ageing, diseases of the body and brain, and an overall decline in the quality of life.
\end{abstract}

Key Words lifestyle-related disease, obesity, autonomic nervous system, brain-derived neurotrophic factor (BDNF)

\section{Etiology of Obesity and Metabolic Syndrome}

Bray (1) has proposed the MONA LISA hypothesis, an acronym for Most Obesities kNown Are Low In Sympathetic Activity indicating that obesity is associated with a relative or absolute reduction in the activity of the thermogenic component of the sympathetic nervous system. Since the $\beta 3$-adrenergic receptor plays a significant role in the control of lipolysis and thermogenesis in brown adipose tissue4 through autonomic nervous system (ANS) activity, we first determined the prevalence of the polymorphism in 204 subjects (2). Results indicated that the subjects with the variant, even the heterozygotes, demonstrated significantly lower resting ANS activity than normal subjects, whereas the clinical characteristics did not differ between groups.

It is not uncommon for people to spend one-half of their waking day sitting, with relatively idle muscles (NEAT: Non-Exercise Activity Thermogenesis). Hamilton et al. (3) have argued that sitting time and NEAT have been linked in epidemiological studies to rates of metabolic syndrome, type 2 diabetes, obesity, and CVD. A longitudinal study by Hancox et al. (4) assessed approximately 1000 unselected individuals born in Dunedin, New Zealand, in 1972-73 at regular intervals up to age $26 \mathrm{y}$. Television viewing in childhood and adolescence is associated with overweight, poor fitness, smoking, and raised cholesterol in adulthood. These data strongly sug-

E-mail: moritani.toshio.6e@kyoto-u.ac.jp gest that excessive TV viewing might have long-lasting adverse effects on health. Furthermore, even when adults meet physical activity guidelines, sitting for prolonged periods can compromise metabolic health. Television time and objective measurement studies show deleterious associations, and breaking up sedentary time is beneficial. Sitting time, TV time, and time sitting in automobiles increase premature mortality risk (5).

There are growing expectations that too much sitting is a real and substantial risk to health. One of the intriguing findings from these accelerometer measurement studies is that breaks in sedentary time were shown to have beneficial associations with metabolic biomarkers $(5,6)$, due possibly to challenging and enhancing the autonomic nervous system, which regulates body weight and appetite $(7,8)$. Thus, NEAT is likely to serve as a crucial thermoregulatory switch between excess energy being stored or dissipated. Determining the physiological regulation of NEAT may lead to a better understanding of the patho-physiology of obesity and improved prevention and treatment of obesity. Our exercise intervention study has also demonstrated a strong possibility that ANS activity can be enhanced by exercise training among previously obese menopausal women as well as school children with reduced ANS activity $(7,9)$.

As far as the pathogenesis of diabetes is concerned, Stuart et al. (10) demonstrated that the limited physical activity dictated by bed rest for as little as seven days is associated with substantial resistance to insulin's effects 
on glucose metabolism. Further, the data suggest that these effects occur primarily in skeletal muscle with little change in insulin action on the liver. Thus we should consider the strong possibility that the primary cause of diabetes rests on inactivity-induced muscle metabolic disorder. Exercise can directly activate glucose uptake in skeletal muscle by inducing translocation of glucose transporters (GLUT-4) to the cell surface via an insulinindependent mechanism. In fact, contraction-stimulated GLUT-4 translocation is not impaired in insulinresistant conditions such as Type 2 diabetes and obesity $(11,12)$.

Recent study also indicates that a defect in the ability to oxidize lipid in skeletal muscle is evident with obesity, which is corrected with exercise training but persists after weight loss (13). In this study, there was no difference in muscle fatty acid oxidation (FAO) between the extremely obese and weight loss groups, and FAO was nearly $45 \%$ depressed compared with the lean subjects. Muscle FAO also did not change in extremely obese women before and $1 \mathrm{yr}$ after a $55-\mathrm{kg}$ weight loss. In contrast, 10 consecutive days of exercise training significantly increased FAO in the skeletal muscle of lean (1.7-fold), obese (1.8-fold), and previously extremely obese subjects after weight loss (2.6-fold). Since this study with muscle biopsy eliminates the influence of autonomic nervous system activity and circulating hormones, the results highlight the vital role of exercise on muscle fat oxidation capacity (13).

\section{Metabolic and Appetite Response of Fat, Protein and Carbohydrate-New Evidence}

Earlier study (14) demonstrated that there was no immediate metabolic response to fat oxidation for nearly $1000 \mathrm{Kcal}$ of excess fat intake. Because fat intake does not promote fat oxidation, excess dietary fat is almost entirely stored in adipose tissue (13). In hypercaloric condition, fat oxidation is inhibited and large lipid storage is demonstrated. The conversion of $\mathrm{CHO}$ into fat is an energy requiring process, in which nearly $25 \%$ of the energy content of carbohydrates is converted into heat. Deposition of dietary triglycerides into adipose tissue requires very little energy $(0-2 \%)$. During $\mathrm{CHO}$ overfeeding, the hepatic denovo lipogenesis would not exceed more than $10 \mathrm{~g}$ fatty acids per day (14). Recent studies (15) have also demonstrated that over $1 \mathrm{y}$, there was a favorable effect of an energy-restricted highcarbohydrate, low-fat diet compared with an isocaloric high-fat, low-carbohydrate diet on mood state and affect in overweight and obese individuals.

Our understanding of how neurohormonal gut-brain signaling regulates energy homeostasis has advanced significantly in recent years $(16,17)$. Ghrelin is an orexigenic peptide produced by the stomach, which appears to act as a meal initiator. Satiety signals derived from the intestine and pancreas include peptide YY, pancreatic polypeptide, glucagon-like peptide 1, oxyntomodulin, and cholecystokinin. Interestingly, fat appears to suppress ghrelin less potently per calorie than carbohydrate or protein. This may, in part, explain the reduced sati- ety and enhanced weight gain associated with high-fat diets. In line with this finding, it has been clearly demonstrated that consuming a low-carbohydrate (approximately $47 \%$ energy) diet is associated with greater likelihood of being overweight or obese among healthy, free living adults. The lowest risk may be obtained by consuming $47 \%$ to $64 \%$ energy from carbohydrates (18). Gut hormone-based therapies may thus provide an effective and well-tolerated treatment for obesity.

Interestingly, Martins et al. (16) investigated the acute effects of exercise on the postprandial levels of appetiterelated hormones and metabolites, energy intake (EI) and subjective measures of appetite. Ghrelin, polypeptide YY (PYY), glucagon-like peptide-1 (GLP-1) and pancreatic polypeptide (PP) were measured in the fasting state and postprandially in 12 healthy, normal weight volunteers using a randomized crossover design. One hour after a standardized breakfast, subjects either cycled for $60 \mathrm{~min}$ at $65 \%$ of their maximal heart rate or rested. Exercise significantly increased mean PYY, GLP-1 and PP levels, and this effect was maintained during the post-exercise period for GLP-1 and PP. No significant effect of exercise was observed on postprandial levels of ghrelin.

In conclusion, acute exercise, of moderate intensity, temporarily decreased hunger sensations and was able to produce a short-term negative energy balance. This impact on appetite and subsequent energy homeostasis was not explained by changes in postprandial levels of ghrelin; however, 'exercise-induced anorexia' may potentially be linked to increased PYY, GLP-1 and PP levels (16). Interestingly, Crum et al. (19) have recently investigated whether physiological satiation as measured by the gut peptide ghrelin may vary depending on the mindset in which one approaches consumption of food. On 2 separate occasions, participants $(n=46)$ consumed a 380-calorie milkshake under the pretense that it was either a 620-calorie "indulgent" shake or a 140-calorie "sensible” shake. Participants' satiety was consistent with what they believed they were consuming rather than the actual nutritional value of what they consumed. Thus, the effect of food consumption on ghrelin may be psychologically mediated, and mindset meaningfully affects physiological responses to food (19).

\section{Exercise, Nutrition and Cognitive Function}

Synaptic plasticity and behaviors are likely dependent on the capacity of neurons to meet the energy demands imposed by neuronal activity. In an area critical for learning and memory, the hippocampus, exercise modified aspects of energy metabolism by decreasing oxidative stress and increasing the levels of cytochrome c oxidase-II, a specific component of mitochondrial machinery (20). While exercise significantly increased the mRNA levels of brain-derived neurotrophic factor (BDNF) in the hippocampus above sedentary levels, pharmacological treatment fully blocked the exerciseinduced effect on BDNF mRNA, effectively reducing them to the mRNA levels of a sedentary state.

The circulating BDNF is reduced in patients with 
major depression and type-2 diabetes. Because acute exercise increases BDNF production in the hippocampus and cerebral cortex, Seifert et al. (21) hypothesized that endurance training would enhance the release of BDNF from the human brain as detected from arterial and internal jugular venous blood samples. In a randomized controlled study, 12 healthy sedentary males carried out 3 mo of endurance training or served as controls. Results indicated that $3 \mathrm{mo}$ of endurance training significantly enhanced the resting release of BDNF $(p<0.05)$, with no significant change in the control subjects.

The hippocampus shrinks in late adulthood, leading to impaired memory and increased risk for dementia. Hippocampal and medial temporal lobe volumes are larger in higher-fit adults, and physical activity training increases hippocampal perfusion, but the extent to which aerobic exercise training can modify hippocampal volume in late adulthood remains unknown. Recent results from an exercise intervention study by Erickson et al. (22) demonstrate, in a randomized controlled trial with 120 older adults, that aerobic exercise training increases the size of the anterior hippocampus, leading to improvements in spatial memory. This study clearly demonstrated that exercise training increased hippocampal volume by $2 \%$, effectively reversing age-related loss in volume by 1 to $2 \mathrm{y}$. In sum, the hippocampus remains plastic in late adulthood and $1 \mathrm{y}$ of aerobic exercise was sufficient for enhancing volume. Increased hippocampal volume translates to improved memory function and higher serum BDNF (22). These results demonstrate that higher fitness levels are protective against loss of hippocampal volume.

New evidence shows that dietary supplementation of DHA and curcumin has important actions on the mechanisms that maintain membrane physiology and neuronal signaling (23). Emerging studies indicate that exercise is capable of boosting the health effects of certain nutrients such as omega-3 fatty acids. Therapies based on DHA, curcumin, and exercise can benefit the brain, and have long-term consequences on molecular systems responsible for maintaining synaptic function, underlying higher order operations such as learning and memory, and emotions (23).

\section{REFERENCES}

1) Bray GA. 1991. Obesity, a disorder of nutrient partitioning: The MONA LISA hypothesis. J Nutr 121: 1146-1162.

2) Shihara N, Yasuda K, Moritani T, et al. 1999. The association between Trp64Arg polymorphism of the beta3adrenergic receptor and autonomic nervous system activity. J Clin Endocrinol Metab 84: 1623-1627.

3) Hamilton MT, Hamilton DG, Zderic TW. 2007. Role of low energy expenditure and sitting in obesity, metabolic syndrome, type 2 diabetes, and cardiovascular disease. Diabetes 56: 2655-2667.

4) Hancox RJ, Milne BJ, Poulton R. 2004. Association between child and adolescent television viewing and adult health: a longitudinal birth cohort study. Lancet
364: 257-262.

5) Healy GN, Dunstan DW, Salmon J, et al. 2008. Breaks in sedentary time: Beneficial associations with metabolic risk. Diabetes Care 31: 661-666.

6) Owen N, Healy GN, Matthews CE, Dunstan DW. 2010. Too much sitting: the population health science of sedentary behavior. Exerc Sport Sci Rev 38: 105-113.

7) Amano M., Kanda T, Ue H, et al. 2001. Exercise training and autonomic nervous system activity in obese individuals. Med Sci Sports Exer 33: 1287-1291.

8) Yoneshiro T, Aita S, Matsushita1 M, et al. 2011. Brown adipose tissue, whole-body energy expenditure, and thermogenesis in healthy adult men. Obesity 19: 13-16.

9) Nagai N., Hamada T., Kimura T. et al. 2004. Moderate physical exercise increases cardiac autonomic nervous system activity in children with low heart rate variability. Child's Nervous System 20: 209-214.

10) Stuart CA, Shangraw RE, Prince MJ, et al. 1988. Bedrest-induced insulin resistance occurs primarily in muscle. Metabolism 37: 802-806.

11) Hayashi T, Hirshman MF, Kurth EJ, et al. 1998. Evidence for 5' AMP-activated protein kinase mediation of the effect of muscle contraction on glucose transport. Diabetes 47: 1369-1373.

12) Moritani T, Kimura T, Hamada T, Nagai N. 2005. Electrophysiology and kinesiology for health and disease. $J$ Electromyogr Kinesiol 15: 240-255.

13) Berggren JR, Boyle KE, Chapman WH, et al. 2009. Skeletal muscle lipid oxidation and obesity: influence of weight loss and exercise. Am J Physiol Endocrinol Metab 294: E726-E732.

14) Jéquier E, Tappy L. 1999. Regulation of body weight in humans. Physiol Rev 79: 451-480.

15) Brinkworth GD, Buckley JD, Noakes M, et al. 2009. Long-term effects of a very low-carbohydrate diet and a low-fat diet on mood and cognitive function. Arch Intern Med 169: 1873-1880.

16) Martins C, Morgan LM, Bloom SR, et al. 2007. Effects of exercise on gut peptides, energy intake and appetite. $J$ Endocrinol 193: 251-258.

17) Wren AM, Bloom SR. 2007. Gut hormones and appetite control. Gastroenterology 132: 2116-2130.

18) Merchant AT, Vatanparast H, Barlas S, et al. 2009. Carbohydrate intake and overweight and obesity among healthy adults. J Am Diet Assoc 109: 1165-1172.

19) Crum AJ, Corbin WR, Brownell KD, Salovey P. 2011. Mind over milkshakes: mindsets, not just nutrients, determine ghrelin response. Health Psychol 30: 424-429.

20) Gomez-Pinilla F, Vaynman S, Ying Z. 2008. Brainderived neurotrophic factor functions as metabotrophin to mediate the effects of exercise on cognition. Eur J Neurosci 28: 2278-2287.

21) Seifert T, Brassard P, Wissenberg M, et al. 2010. Endurance training enhances BDNF release from the human brain. Am J Physiol Regul Integr Comp Physiol 298: R372-R377.

22) Erickson KI, Voss MW, Prakash RS, et al. 2011. Exercise training increases size of hippocampus and improves memory. PNAS 108(7): 3017-3022.

23) Gomez-Pinilla F. 2011. The combined effects of exercise and foods in preventing neurological and cognitive disorders. Preventive Med 52: S75-S80. 